\title{
Oncologic Outcome after Cessation or Dose Reduction of Capecitabine in Patients with Colon Cancer
}

\author{
Jung-A Yun, Hee Cheol Kim, Hyun-Sook Son ${ }^{1}$, Hyoung Ran Kim, Hae Ran Yun, Yong Beom Cho, \\ Seong Hyeon Yun, Woo Yong Lee, Ho-Kyung Chun \\ Department of Surgery, Samsung Medical Center, Sungkyunkwan University School of Medicine, Seoul, \\ ${ }^{1}$ Department of Nursing, Colorectal Cancer Center, Samsung Medical Center, Seoul, Korea
}

Purpose: Oral capecitabine has been used as adjuvant therapy for colorectal cancer patients since the 1990s. Patient-initiated cessation or reduced use of capecitabine occurs widely for various reasons, yet the consequences of these actions are unclear. The present study sought to clarify treatment outcomes in such patients.

Methods: The study included 173 patients who had been diagnosed with stage II or III colon cancer according to the pathologic report after radical surgery at Samsung Medical Center from May 2005 to June 2007 and who had received capecitabine as adjuvant therapy. The patients were divided into groups according to whether the dose was reduced (I, dose maintenance; II, dose reduction) or stopped (A, cycle completion; B, cycle cessation). Recurrence and disease-free survival rates between the two groups each were analyzed.

Results: Of the 173 patients, 128 (74.6\%) experienced complications, most frequently hand-foot syndrome $(\mathrm{n}=114)$. Reduction $(n=35)$ or cessation $(n=18)$ of medication was most commonly due to complications. Concerning reduced dosage, both groups displayed no statistically significant differences in recurrence rate and 3-year disease-free survival rate. Concerning discontinued medication use, the cycle completion group showed an improved recurrence rate $(\mathrm{P}=0.048)$ and 3-year disease-free survival rate $(\mathrm{P}=0.028)$.

Conclusion: The results demonstrate that maintaining compliance with capecitabine as an adjuvant treatment for colon cancer to preventing complications positively affects patient prognosis.

Keywords: Colon cancer; Capecitabine; Dose; Cycle; Disease-free survival

\section{INTRODUCTION}

Colon cancer is the second most common type of cancer worldwide $[1,2]$. About 1 million patients are diagnosed each year, and approximately half of them die of this disease. Also, about 230 thousand patients who undergo colon cancer surgery require adjuvant chemotherapy $[3,4]$.

The most important prognostic factor of colon cancer is path-

Received: March 16, 2010 Accepted: June 27, 2010

Correspondence to: Hee Cheol Kim, M.D.

Department of Surgery, Samsung Medical Center, 50 Irwon-dong,

Gangnam-gu, Seoul 135-710, Korea

Tel: +82-2-3410-1655, Fax: +82-2-3410-6980

E-mail: hckim@skku.edu

(C) 2010 The Korean Society of Coloproctology

This is an open-access article distributed under the terms of the Creative Commons Attribution NonCommercial License (http://creativecommons.org/licenses/by-nc/3.0) which permits unrestricted noncommercial use, distribution, and reproduction in any medium, provided the original work is properly cited. ological stage. Patients diagnosed with stage III based on pathological examination of biopsy specimens have a $67 \% 5$-year survival rate. In patients with stage IV diagnosis, the 5-year survival rate is $30 \%$ if complete excision of metastastics lesions is executed [5]. There are different opinions on the principles of postoperative adjuvant chemotherapy. Stage II or III is often taken as an indication for adjuvant chemotherapy.

With the development of numerous anticancer drugs such as 5-FU, leucovorin, oxaliplatin, irinotecan, TS-1, and capecitabine, adjuvant chemotherapy for colon cancer has been diversified since the 1990s. From a clinical study presented in 2005, capecitabine in 1,987 patients with stage III colon cancer did not show inferior survival rate compared to 5-FU injection. Moreover, it showed fewer side effects than 5-FU [6-8]. Capecitabine is an oral anticancer drug that is easy to take at home, that can eliminate difficulty in finding blood vessels and that can minimize the number of hospital visits.

Although capecitabine has less diarrhea, stomatitis, nausea, 
hair loss, and neutropenia than 5-FU with leucovorin, it causes more hand-foot syndrome [7]. Because of this complication, interruption of medication, depending on an individual's compliance, is frequent. In spite of this problem, little research has been done about whether reduced or interrupted medication affects the prognosis for the disease or not. Therefore, this research was conducted to analyze the effect of dose reduction and interruption of postoperative adjuvant capecitabine on the oncological results of colon cancers.

\section{METHODS}

From May 2005 to June 2007, the number of patients prescribed with capecitabine as postoperative adjuvant chemotherapy for colon cancer at Samsung Hospital (Sungkyunkwan University School of Medicine) was 209. Among the patients, one hundred seventy-three who underwent a radical resection, were diagnosed with stage II or III cancer, and were prescribed with capecitabine as adjuvant chemotherapy were enrolled as the subject group. Patients with rectal cancer or with a relapse during medical treatment were excluded from the study. The final follow-up point was December 2009, and the average followup period was 40 months (range, 30 to 55 months).

Capecitabine was used at $1,250 \mathrm{mg} / \mathrm{m}^{2}$ twice a day for 14 days and was not administered for the next 7 days. This three-week period was considered as one cycle, and a total of 8 cycles was set as a standard. Depending on the stage of hand-food syndrome, patients with grade. I cancer were treated with maintenance of dose and skin protection cream, and patients with grade II or higher cancer were treated with ointment, symptomatic treatment, and reduction of capecitabine dose to $75 \%$ or interruption of medication, depending on the individual's condition. Complications other than hand-foot syndrome, such as diarrhea, stomachache, stomatitis, or blood test abnormality, were treated with either reduced dose or interruption of medication, depending on the practitioner's decision. Categorization of each complication stages was done according to the National Cancer Institute of Canada Common Toxicity Criteria (NCIC) guideline.

One hundred seventy-three patients were categorized into the A group (medication cycle completed) or the B group (medication interrupted), depending on whether the medication was interrupted or not. The patients were also categorized into group I (medicated with full dose) and group II (medicated with reduced dose), depending on the dosage of capecitabine. Medication interruption was defined as cases in which the first 8-period cycle was not completed (medicated only 1 to 7 cycles). Clinical and pathological characteristics, the relapse rate, and the disease-free survival rate were analyzed in each group.

All the data were collected retrospectively through medicalrecord analyses and phone interviews. PASW ver. 17.0 (SPSS Inc., Chicago, IL, USA) was used as the statistics program. The chi-square test was used for analyzing the data, and the KaplanMeier method was used to analyze the relapse and the diseasefree survival rates. Statistical results were considered as significant when the P-value was less than 0.05 .

\section{RESULTS}

\section{Clinicopathological and relapse patterns}

Among the 173 patients, 114 were male and 59 were female. The average age was 57.6 years (range, 29 to 78 years), $114 \mathrm{pa-}$ tients were younger than 65 , and 59 patients were equal to or older than 65 . Thirty-eight patients (22\%) were in stage II, and 135 patients $(78 \%)$ were in stage III. Fifty patients (28.9\%) had a lesion on their right colon, and the other 123 patients (71.1\%) had a lesion on their left colon. One hundred sixty patients $(92.5 \%)$ had an adenocarcinoma, 11 patients $(6.4 \%)$ had mucinous carcinoma, and 2 patients $(1.1 \%)$ had a signet ring cell carcinoma. Fifty-five patients (31.8\%) out of the 173 had lymphatic or venous invasion, 96 patients (55.5\%) did not have such invasion, and in 22 patients (12.7\%), such invasion could not be verified.

During the postoperative follow-up period, physical examination, colonoscopy, CT scanning, and MRI scanning were done in order to find local relapses or remote metastases. Among the 173 patients, twenty-one (12.1\%) had a relapse during observation period. Nine of them (42.9\%) had liver metastasis, 5 of them (22.8\%) had a local relapse, 4 of them (19\%) had lymph node metastasis, 2 of them (9.5\%) had lung metastasis, and 2 of them (9.5\%) had peritoneal dissemination. Two patients (9.5\%) died during the observation period.

\section{Side effects of capecitabine}

Among the 173 patients, 129 (74.6\%) had side effects, and hand-foot syndrome was the most common at $65.9 \%$ (in 114

Table 1. Treatment-related adverse events ${ }^{\mathrm{a}}$

\begin{tabular}{|c|c|c|c|c|}
\hline \multirow[b]{2}{*}{ Complication } & \multirow[b]{2}{*}{ No } & \multicolumn{3}{|c|}{ Yes } \\
\hline & & $\begin{array}{c}\text { Grage } \\
\text { I, II }\end{array}$ & $\begin{array}{c}\text { Grade } \\
\text { II, IV }\end{array}$ & $\begin{array}{l}\text { Undeter- } \\
\text { mined }^{\mathrm{b}}\end{array}$ \\
\hline Hand-foot syndrome & $59(34.1)$ & $84(48.5)$ & $18(10.4)$ & $12(6.9)$ \\
\hline Anorexia/nausea/vomiting & $149(86.1)$ & $13(7.5)$ & $1(0.6)$ & $10(5.8)$ \\
\hline Laboratory abnormalities & $159(91.9)$ & $14(8.1)$ & $0(0)$ & $0(0)$ \\
\hline Diarrhea & $161(93.1)$ & $4(2.3)$ & $2(1.2)$ & $6(3.5)$ \\
\hline Stomatitis & $162(93.6)$ & $11(6.4)$ & $0(0)$ & $0(0)$ \\
\hline Abdominal pain & $168(97.1)$ & $0(0)$ & $0(0)$ & $5(2.9)$ \\
\hline Fatigue or asthenia & $172(99.4)$ & $0(0)$ & $0(0)$ & $1(0.6)$ \\
\hline Alopecia & $172(99.4)$ & $0(0)$ & $0(0)$ & $1(0.6)$ \\
\hline
\end{tabular}

Values are presented as number (\%).

${ }^{a}$ National Cancer Institute of Canada Common Toxicity Criteria were used except for hand-foot syndrome; ' There is no specified description on the chart. 
patients). Reduction or interruption of capecitabine medication was considered if the patient was in grade II or higher hand-foot syndrome. Forty-five (39.5\%) among 114 patients were in grade II or higher hand-foot syndrome. Twenty-one (20.2\%) among 129 patients with grade III or above had side effects (Table 1).

\section{Cause of medication interruption or reduction}

Among 46 patients with interrupted or reduced medication, 35 (76.1\%, group II) had reduced dosage (75\%). In seven (15.2 $\%)$ of those 35 patients the medication was eventually interrupted, for a total of 18 patients (39.1\%, group B) with interrupted medication. Forty-two of the 46 patients $(91.3 \%)$ had interrupted or reduced medication because of severe side effects, and the other 4 patients $(8.7 \%)$ had interrupted medication because of poor compliance. Among the former 42 patients with interrupted or reduced dosage, 39 (92.9\%) had hand-foot syndrome, $12(28.6 \%)$ had nausea, 8 (19.0\%) had diarrhea, 7 (16.7\%) had abnormal blood test results, 3 (7.1\%) had neutropenia, $3(7.1 \%)$ had hyperbilirubinemia, 4 (9.5\%) had cheilitis, and $2(4.8 \%)$ had stomachache.

Relapse and disease-free survival rates by dosage reduction Patients without dosage reduction were in group I $(n=138$,

Table 2. Characteristics of patients in Group I, dose maintenance, and Group II, dose reduction

\begin{tabular}{lccc}
\hline Characteristics & Group I $(\mathrm{n}=138)$ & Group II $(\mathrm{n}=35)$ & P-value \\
\hline Sex & & & \\
$\quad$ Male & $92(66.7)$ & $22(62.9)$ & 0.693 \\
Female & $46(33.3)$ & $13(37.1)$ & \\
Age & & & \\
$\quad<65$ & $96(69.6)$ & $18(51.4)$ & 0.048 \\
$\geq 65$ & $42(30.4)$ & $17(48.6)$ & \\
Location & & & \\
$\quad$ Rt. colon & $40(29)$ & $10(28.6)$ & 1.000 \\
Lt. colon & $98(71)$ & $25(71.4)$ & \\
Stage & & & \\
II & $31(22.5)$ & $7(20)$ & 0.823 \\
III & $107(77.5)$ & $28(80)$ & \\
Pathologic type & & & \\
Adeno carcinoma & $127(92)$ & $33(94.3)$ & 1.000 \\
Mucinous carcinoma/signet & $11(8)$ & $2(5.7)$ & \\
$\quad$ ring cell carcinoma & & & \\
Complication & & & \\
Yes & & & \\
No & $94(68.1)$ & $35(100)$ & $<0.01$ \\
Recurrence & $44(31.9)$ & $0(0)$ & \\
Yes & & & \\
No & $14(10.1)$ & $7(20)$ & 0.144 \\
\hline
\end{tabular}

Values are presented as number (\%).

Rt. colon, from ascending to mid-transverse colon; Lt. colon, from distal transverse to sigmoid colon.
$79.8 \%$ ), and patients with reduced dosage were in group II ( $\mathrm{n}=$ $35,20.2 \%$ ), and there were no statistically significant differences in sex, location of lesion, pathological stages, and pathological characteristics, but group II has relatively older patients ( $\mathrm{P}=$ 0.048). There was no significant difference in relapse rate between the two groups $(\mathrm{P}=0.144)$ (Table 2). The three-year disease-free survival rate of group I was $90.5 \%$, and that of group II was $82.4 \%$, but this difference was not statistically significant $(\mathrm{P}=0.126)$ (Fig. 1).

\section{Relapse and disease-free survival rates by medication interruption}

Patients who had completed 8 cycles of medication were in group A ( $\mathrm{n}=155)$, and patients who had interrupted medication were in group B $(\mathrm{n}=18)$. There were no statistically significant differences in sex, age, location of lesion, pathological stages, pathological characteristics, and side effect occurrence (Table 3). However, the relapse rates of the two groups were $10.3 \%$ and $27.8 \%$, respectively, and this difference was statistically significant $(\mathrm{P}=0.048)$. The three-year disease-free survival rates of the two groups were $90.7 \%$ and $70.9 \%$, respectively, again this difference was statistically significant $(P=0.028)$ (Fig. 2).

Table 3. Characteristics of patients in Group A, cycle completion, and Group B, cycle cessation

\begin{tabular}{lrrr}
\hline Characteristics & Group A ( $\mathrm{n}=155)$ & Group B (n=18) & P-value \\
\hline Sex & & & \\
$\quad$ Male & $105(67.7)$ & $9(50)$ & 0.188 \\
$\quad$ Female & $50(32.3)$ & $9(50)$ & \\
Age & & & \\
$\quad<65$ & $105(67.7)$ & $9(50)$ & 0.188 \\
$\geq 65$ & $50(32.3)$ & $9(50)$ & \\
Location & & & \\
$\quad$ Rt. colon & $42(27.1)$ & $8(44.4)$ & 0.168 \\
Lt. colon & $113(72.9)$ & $10(55.6)$ & \\
Stage & & & \\
II & $33(21.3)$ & $5(27.8)$ & 0.551 \\
III & $122(78.7)$ & $13(72.2)$ & \\
Pathologic type & & & \\
Adeno carcinoma & $142(91.6)$ & $18(100)$ & 0.366 \\
Mucinous carcinoma/signet & $13(8.4)$ & $0(0)$ & \\
$\quad$ ring cell carcinoma & & & \\
Complication & & & \\
$\quad$ Yes & $113(72.9)$ & $16(88.9)$ & 0.165 \\
No & $42(27.1)$ & $2(11.1)$ & \\
Recurrence & & & \\
Yes & $16(10.3)$ & $5(27.8)$ & 0.048 \\
No & $139(89.7)$ & $13(72.2)$ & \\
\hline
\end{tabular}

Values are presented as number (\%).

Rt. colon, from ascending to mid-transverse colon; Lt. colon, from distal transverse to sigmoid colon. 


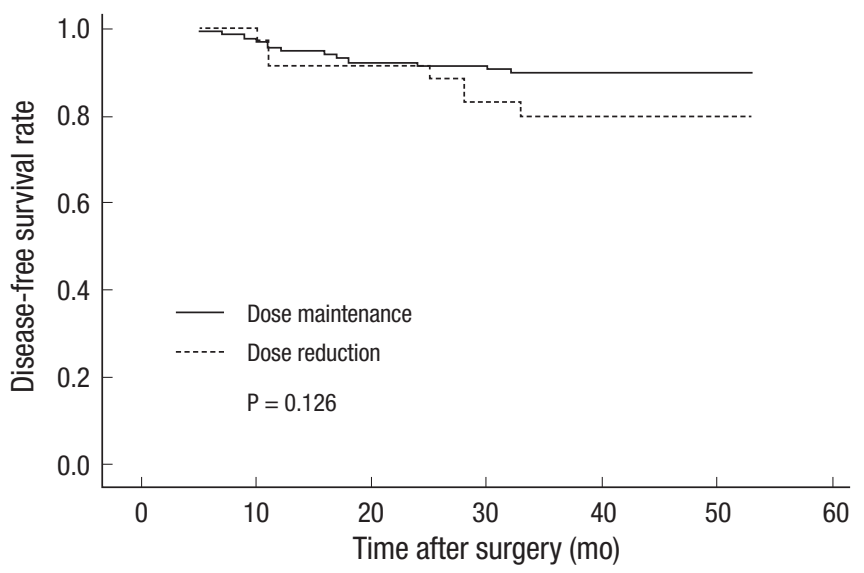

Fig. 1. Disease-free survival rates for dose maintenance and dose reduction.

\section{DISCUSSION}

Capecitabine is a $5-\mathrm{FU}$ prodrug which is converted into 5-FU by thymidine phosphorylase inside the cancer tissue. The concentration of thymidine phosphorylase in cancer cells is higher than that in normal cells; thus, cancer cells are affected more selectively [9]. The maximum concentration in the blood is reached in $1.5-2$ hours when $1,250 \mathrm{mg} / \mathrm{m}^{2}$ (standard amount) is taken $[10,11]$. Capecitabine intake for postoperative adjuvant purposes is known to be safer while having effects similar to those of other intravenous anticancer drugs [6-8]. The benefits of capecitabine are ease of oral administration, no need for reserved blood vessel, and home administration of the drug. Also, there are reports that it is less expensive than administrating 5-FU and other intravenous anticancer drugs $[10,12,13]$. However, there are disadvantages in capecitabine administration as well. First, it is difficult to monitor whether the patient is taking the drug in the right way because it is often administered at the patient's home, and the result is greatly affected by patient's compliance. Second, although it has fewer occurrences of diarrhea, stomatitis, nausea, hair loss, and neutropenia, it has more occurrences of hand-foot syndrome [7]. In cases involving the side effects of capecitabine where the dosage is reduced or medication is interrupted, there have been few discussions about how the reduction and/or interruption affects the prognosis. However, there are reports that in older patients with breast cancer, a lower dosage of capecitabine shows effects similar to those achieved with normal dosage $[14,15]$. In addition, there has been a case study where a patient with stage IV gastric cancer had a reduced dosage because of severe hematological side effects, but was completely recovered [16]. However, reduced dosage in older patients with non-Hodgkin lymphoma resulted in relapse and exacerbation, which increased mortality [17]. Since dosage reduction is done in older patients in order to reduce complications, further research is nec-

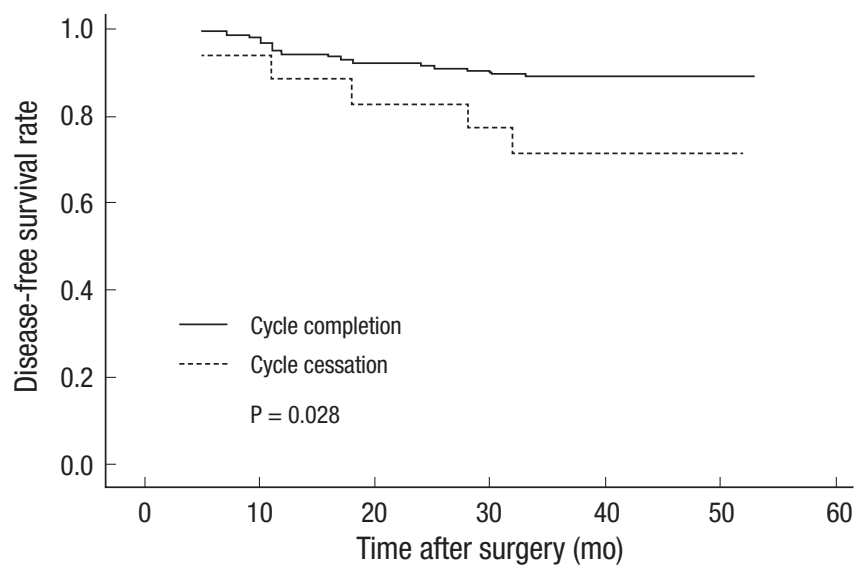

Fig. 2. Disease-free survival rates for cycle completion and cycle cessation.

essary to establish the relation between prognosis and dose reduction.

This research was done with 173 colon cancer patients at Samsung Medical Center who took capecitabine as a postoperative adjuvant treatment. It was done in order to see whether reduced dosage or interrupted medication affected the relapse rate and the prognosis. In cases where dosage was reduced, there were no statistically significant differences in the relapse rate and the disease-free survival rate. In cases where medication was interrupted completely, the relapse rate was higher, and the disease-free survival rate was lower. However, there are several disputes about whether the difference in the prognosis was solely affect ed by the reduced dosage or interrupted medication. First, the number of patients was relatively small, which caused a low number of relapsed patients and difficulty with subject group categorization. This research only separated the patients into two groups based on their medication period (8-cycle scompletion). Among these patients, there were $6 \mathrm{pa}$ tients who took the drug for less than 4 cycles. There are no data about how capecitabine affects oncological change when administered for such a short period. Also, analysis of oncological outcome based on detailed categorization of administration period was difficult in this research, as was finding the relationship between reduced dosage and prognosis. Secondly, there are factors such as age, physical status, and compliance that can affect the oncological outcome and are difficult to analyze. There were no clinicopathological differences between the interrupted medication group and the completed medication group. However, the reduced dosage group turned out to have older patients, an intrinsic error of the retrospective study. This might have affected the oncological results and the medication side effects. In the future, prospective data collection and analysis of various factors that can affect prognosis are necessary. Lastly, a patient with short follow-up period (32 months) was included in the subject group. This patient might have had 
a relapse if the follow-up had been longer, so the relapse rate and the disease-free survival rate might be underestimated.

Interrupted medication or reduced dosage was most common when complications occurred. One hundred twenty-nine (74.6 $\%)$ out of 173 patients had complications, but other research has reported complications of $22-31 \%[6,7]$. The most common complication was hand-foot syndrome, for which the incidence was $65.9 \%$ (114 patients), which is very high when compared with other reports $(6 \%)[6,7]$. Stricter judgment and over measurement are thought to be the cause of this high frequency. There could have been error by prepossession of the researcher, and it was difficult to collect accurate data for patients without a detailed description. Also, the amount of capecitabine was based on accepted dosage for Westerners and could have been an overdose for Asians.

The most frequent cause of interrupted medication or reduced dosage was occurrence of complications. In case of cetuximab, there is a report that occurrence of skin disease is a prognostic factor that contributes to patient's outcome $[18,19]$. While this situation might be true in the case of capecitabine, there are no reports on this topic. Accordingly, more research on the complications of capecitabine, which cause dosage reduction or interrupted medication and on the effects of reduction printerruption of medication on the prognosis is needed.

Capecitabine is one of the drugs that can be used with ease in colon cancer patients for postoperative adjuvant purposes. The most common cause that induces interrupted medication or dosage reduction is development of complications. This research suggests that interrupted medication affects the relapse rate whereas reduced dosage does not. Accordingly, minimizing the toxicity of the drugs and finishing the scheduled treatment are thought to result in improved prognosis. Therefore, continuous treatment, prevention of complications, and active and systematic treatment are necessary.

\section{CONFLICT OF INTEREST}

No potential conflict of interest relevant to this article was reported.

\section{REFERENCES}

1. Jemal A, Siegel R, Ward E, Hao Y, Xu J, Thun MJ. Cancer statistics, 2009. CA Cancer J Clin 2009;59:225-49.

2. Center MM, Jemal A, Smith RA, Ward E. Worldwide variations in colorectal cancer. CA Cancer J Clin 2009;59:366-78.

3. Ayanian JZ, Zaslavsky AM, Fuchs CS, Guadagnoli E, Creech CM, Cress $\mathrm{RD}$, et al. Use of adjuvant chemotherapy and radiation therapy for colorectal cancer in a population-based cohort. J Clin Oncol 2003;21:1293-300.

4. Benson AB 3rd, Schrag D, Somerfield MR, Cohen AM, Figueredo AT, Flynn PJ, et al. American Society of Clinical Oncology recommendations on adjuvant chemotherapy for stage II colon cancer. J Clin Oncol 2004;22:3408-19.

5. Tomlinson JS, Jarnagin WR, DeMatteo RP, Fong Y, Kornprat P, Gonen M, et al. Actual 10-year survival after resection of colorectal liver metastases defines cure. J Clin Oncol 2007;25:4575-80.

6. Scheithauer W, McKendrick J, Begbie S, Borner M, Burns WI, Burris HA, et al. Oral capecitabine as an alternative to i.v. 5-fluorouracil-based adjuvant therapy for colon cancer: safety results of a randomized, phase III trial. Ann Oncol 2003;14:1735-43.

7. Twelves C, Wong A, Nowacki MP, Abt M, Burris H 3rd, Carrato A, et al. Capecitabine as adjuvant treatment for stage III colon cancer. N Engl J Med 2005;352:2696-704.

8. Lamberti C, Sauerbruch T, Glasmacher A. Adjuvant capecitabine is at least as effective as fluorouracil plus leucovorin for survival in people with resected stage III colon cancer. Cancer Treat Rev 2005;31:648-52.

9. Schuller J, Cassidy J, Dumont E, Roos B, Durston S, Banken L, et al. Preferential activation of capecitabine in tumor following oral administration to colorectal cancer patients. Cancer Chemother Pharmacol 2000;45:291-7.

10. Eggington S, Tappenden P, Pandor A, Paisley S, Saunders M, Seymour M, et al. Cost-effectiveness of oxaliplatin and capecitabine in the adjuvant treatment of stage III colon cancer. Br J Cancer 2006;95:1195-201.

11. Ishikawa T, Utoh M, Sawada N, Nishida M, Fukase Y, Sekiguchi F, et al. Tumor selective delivery of 5-fluorouracil by capecitabine, a new oral fluoropyrimidine carbamate, in human cancer xenografts. Biochem Pharmacol 1998;55:1091-7.

12. Douillard JY, Tilleul P, Ychou M, Dufour P, Perrocheau G, Seitz JF, et al. Cost consequences of adjuvant capecitabine, Mayo Clinic and de Gramont regimens for stage III colon cancer in the French setting. Oncology 2007;72:248-54.

13. Shiroiwa T, Fukuda T, Shimozuma K, Ohashi Y, Tsutani K. Costeffectiveness analysis of capecitabine compared with bolus 5-fluorouracil/l-leucovorin for the adjuvant treatment of colon cancer in Japan. Pharmacoeconomics 2009;27:597-608.

14. Bajetta E, Procopio G, Celio L, Gattinoni L, Della Torre S, Mariani $\mathrm{L}$, et al. Safety and efficacy of two different doses of capecitabine in the treatment of advanced breast cancer in older women. J Clin Oncol 2005;23:2155-61.

15. Honma H. Successful treatment of an elderly patient with pretreated recurrent breast cancer using low-dose capecitabine. Gan To Kagaku Ryoho 2006;33:2045-8.

16. Miyazaki Y, Imamura H, Kishimoto T, Furukawa H, Ota K, Tatsuta M. A case of unresectable advanced gastric cancer successfully treated with continuous S-1 + CPT-11 chemotherapy accompanied by dose reduction against grade 4 hematological adverse event. Gan To Kagaku Ryoho 2008;35:2060-2.

17. Sonneveld P, Huijgens PC, Hagenbeek A. Dose reduction is not recommended for elderly patients undergoing chemotherapy for non-Hodgkin lymphoma. Ned Tijdschr Geneeskd 1999;143:418-9.

18. Gamucci T, Nelli F, Cianci G, Grassi G, Moscetti L, Sperduti I, et 


\section{Coloproctology Jung-A Yun, et al.}

al. A phase II study of cetuximab/irinotecan in patients with heavily pretreated metastatic colorectal cancer: predictive value of early specific toxicities. Clin Colorectal Cancer 2008;7:273-9.

19. Orditura M, De Vita F, Galizia G, Lieto E, Vecchione L, Vitiello F, et al. Correlation between efficacy and skin rash occurrence following treatment with the epidermal growth factor receptor inhibitor cetuximab: a single institution retrospective analysis. Oncol Rep 2009;21:1023-8. 